\section{Aspectos nutricionais associados à infecção crônica pelo Trypanosoma cruzi (Chagas 1909) entre idosos: Projeto Bambuí}

\author{
Nutritional aspects associated with chronic \\ Trypanosoma cruzi (Chagas 1909) infection \\ among older adults: Bambuí Project
}

\section{Aspectos nutricionales asociados a la infección crónica por el Trypanosoma cruzi (Chagas 1909) entre ancianos: Proyecto Bambuí}

João Paulo dos Santos 1 Maria Fernanda Lima-Costa 2

Sérgio Viana Peixoto 2,3

\footnotetext{
${ }^{1}$ Centro de Pesquisas René Rachou, Fundação Oswaldo Cruz, Belo Horizonte, Brasil. 2 Núcleo de Estudos em Saúde Pública e Envelhecimento, Fundação Oswaldo Cruz/Universidade Federal de Minas Gerais, Belo Horizonte, Brasil. 3 Escola de Enfermagem, Universidade Federal de Minas Gerais, Belo Horizonte, Brasil.

Correspondência S. V. Peixoto

Escola de Enfermagem, Universidade Federal de Minas Gerais. Av. Alfredo Balena 190, Belo Horizonte, MG 30130-100 Brasil.

sergio@cpqrr.fiocruz.br
}

\section{Abstract}

The aim of the study was to verify nutritional aspects associated with chronic Trypanosoma cruzi infection among baseline participants from the Bambui cohort study on aging. The analysis included $84.9 \%(1,479)$ of residents of Bambui, Minas Gerais State, Brazil, who were 60 years or older in 1997. T. cruzi infection was investigated by three serological tests, and nutritional status was assessed by anthropometric and biochemical variables. Associations were evaluated by prevalence ratios and confidence intervals (95\%CI) using Poisson regression. T. cruzi infection was present in $38.1 \%$ of patients. All anthropometric variables were significantly associated with infection, showing lower values among patients with positive serology. No biochemical variables were associated with infection. The results showed the coexistence of chronic Chagas disease and poor nutritional status in the study population, reinforcing the importance of nutritional evaluation among elderly people presenting chronic T. cruzi infection.

Chagas Disease; Nutrition; Aged

\section{Resumo}

O objetivo do estudo foi verificar os aspectos nutricionais associados à infecção crônica pelo Trypanosoma cruzi entre os participantes da linha de base da coorte de idosos de Bambuí, Minas Gerais, Brasil. A análise incluiu 84,9\% (1.479) de todos os residentes com 60 anos ou mais na cidade em 1997. A infecção pelo Tr. cruzi foi avaliada por três testes sorológicos e o perfil nutricional foi caracterizado por variáveis antropométricas e bioquímicas. As associações foram avaliadas pelas razões de prevalência e intervalos de 95\% de confiança, utilizando a regressão de Poisson robusta e ajustando por potenciais fatores de confusão. A infecção foi observada em $38,1 \%$ dos idosos. Todas as variáveis antropométricas apresentaram associação significativa com a infecção, evidenciando menores valores entre os idosos com sorologia positiva. As variáveis bioquímicas não foram associadas ao evento estudado. Os resultados evidenciaram a concomitância da doença de Chagas crônica e pior estado nutricional nessa população, reforçando a importância da avaliação nutricional entre idosos com infecção crônica pelo Tr. cruzi.

Doença de Chagas; Avaliação Nutricional; Idoso 


\section{Introdução}

A doença de Chagas é reconhecida pela Organização Mundial da Saúde (OMS) como uma das 17 doenças tropicais mais negligenciadas do mundo, constituindo como um problema de saúde pública, sobretudo na América Latina 1. Nessa região, estima-se que existam 28 milhões de pessoas sob o risco de contrair a infecção e entre 8 e 10 milhões de indivíduos infectados e que ocorram mais de 10 mil óbitos anuais 2,3,4,5. Devido a movimentos migratórios, casos novos da doença têm sido notificados em países não endêmicos, como Estados Unidos, Canadá, Espanha e Suíça, associados à transmissão congênita e/ou transfusional, tornando-se um problema emergente também nessas regiões 3,6.

No Brasil, as primeiras ações públicas de controle objetivaram o combate de populações intradomiciliares do Triatoma infestans 7 , principal vetor no país, e foram responsáveis pela redução da transmissão do Trypanosoma cruzi ao homem ${ }^{8}$. No entanto, por conta do efeito coorte, o impacto dessa doença para populações idosas que viveram em áreas endêmicas é cada vez mais evidente. A análise das taxas de mortalidade (1980-1995) e de internações hospitalares (1992-2000) pelo Sistema Único de Saúde (SUS) para doença de Chagas mostrou um aumento dos coeficientes com o aumento da idade para a população brasileira 9 . Nesse sentido, e considerando o aumento da população idosa observada no Brasil e no mundo, o entendimento de como a doença de Chagas se associa às demais condições de saúde dos idosos, incluindo os fatores nutricionais, torna-se relevante para um adequado planejamento de ações direcionadas a tal população.

Em recente revisão sobre aspectos nutricionais e doenças negligenciadas no Brasil, fica evidente a escassez de estudos que exploraram essa associação para doença de Chagas, sobretudo os de base populacional 10. A maioria dos estudos existentes foi desenvolvida em populações selecionadas, tais como pacientes atendidos no âmbito hospitalar ou ambulatorial, com exceção para resultados já publicados do Projeto Bambuí, que evidenciou associação significativa entre baixo peso e infecção pelo Tr. cruzi entre idosos 11 .

O objetivo do presente estudo foi investigar a associação entre estado nutricional, definido por parâmetros antropométricos e bioquímicos, e a infecção crônica pelo Tr. cruzi, considerando o ajuste por potenciais fatores de confusão, em um estudo de base populacional conduzido entre idosos residentes na cidade de Bambuí, Minas Gerais, Brasil. Um objetivo adicional foi verificar se essa associação se modifica em diferentes faixas etárias.

\section{Métodos}

\section{Projeto Bambuí}

O Município de Bambuí se situa na mesorregião centro-oeste do Estado de Minas Gerais, a 215km da capital Belo Horizonte. A extensão de sua área é de $1.455,82 \mathrm{~km}^{2}$, caracterizada pela economia agrícola. Na época de realização do estudo, o município possuía cerca de 20.500 habitantes, sendo $73 \%$ residentes na zona urbana 12.

Os participantes da linha de base da coorte de idosos de Bambuí foram identificados por censo completo realizado na comunidade em 1996. Todos os 1.742 residentes na área urbana com 60 ou mais anos de idade em 1o de janeiro de 1997 foram selecionados para participar da linha de base do estudo de coorte. Desses, 1.606 (92,2\%) foram entrevistados e $1.496(85,9 \%)$ realizaram exames clínicos e laboratoriais.

A coorte de idosos de Bambuí foi aprovada pelo Comitê de Ética em Pesquisa da Fundação Oswaldo Cruz (Fiocruz), e todos os participantes assinaram um termo de consentimento livre e esclarecido.

\section{Variáveis estudadas}

As informações para este estudo foram obtidas por meio do questionário Bambuí Health and Aging Study (BHAS), de exame clínico e coleta de sangue. A avaliação antropométrica, medida de pressão arterial e coleta de amostras de sangue para análises bioquímicas foram realizadas na clínica de campo do projeto. Para os exames bioquímicos, os participantes foram orientados a realizar jejum mínimo de 12 horas. As amostras foram centrifugadas, refrigeradas e, posteriormente, encaminhadas ao Laboratório de Epidemiologia e Antropologia Médica do Centro de Pesquisas René Rachou/Fiocruz em Belo Horizonte ${ }^{12}$.

A variável dependente do estudo foi a infecção pelo Tr. cruzi, avaliada por três diferentes testes sorológicos: o ensaio de hemaglutinação (Biolab Mérieux, Rio de Janeiro, Brasil) e dois testes imunoenzimáticos (ELISA) (Abbott Laboratories, Inc., Chicago, Estados Unidos; e Wiener Laboratories, Rosario, Argentina). A infecção foi definida pela sorologia positiva em todos os três exames e a ausência de infecção quando todos os resultados foram negativos, mas 17 idosos foram excluídos das análises pela discordância de resultados. 
As medidas antropométricas foram realizadas por técnicos treinados, utilizando-se equipamentos-padrão (CMS Weighing Equipment Ltd., Londres, Reino Unido). Peso (kg), estatura (m), circunferência da cintura (CC), dobra cutânea tricipital (DCT) e perímetro do braço (PB) foram aferidos com os idosos usando roupas leves e descalços, de acordo com as técnicas preconizadas na literatura 13. Para o peso, utilizou-se uma balança eletrônica digital portátil, com capacidade de $150 \mathrm{~kg}$ e precisão de $50 \mathrm{~g}$. A estatura foi obtida com os indivíduos na posição vertical, utilizando-se estadiômetro de alumínio com capacidade de $2 \mathrm{~m}$ e $0,1 \mathrm{~cm}$ de precisão. $\mathrm{O}$ índice de massa corporal (IMC) foi calculado como o peso (kg) dividido pelo quadrado da altura (m).

A CC e o PB foram aferidos com uso de uma fita métrica flexível e inelástica, com o entrevistado de pé. Na medida da CC, a fita circundou o indivíduo no ponto médio entre a última costela e crista ilíaca. A DCT foi avaliada por meio de compasso científico com escala de 0 a $65 \mathrm{~mm}$, precisão de $1 \mathrm{~mm}$ e pressão constante da mola de $10 \mathrm{~g} / \mathrm{mm}^{3}$ em toda faixa de abertura. Com base nas medidas da DCT e do PB, realizou-se a estimativa da circunferência muscular do braço (CMB), sendo 14:

$\mathrm{CMB}(\mathrm{cm})=[\mathrm{PB}(\mathrm{cm})-(\pi \mathrm{x}$ DCT $(\mathrm{cm}))]$

Para o cálculo da área muscular do braço corrigida $(\mathrm{AMBc})$, foram utilizadas as seguintes equações 15:

Homem: $\mathrm{AMBc}\left(\mathrm{cm}^{2}\right)=\frac{[\mathrm{PB}(\mathrm{cm})-(\pi \times \mathrm{DCT}(\mathrm{cm}))]^{2}}{4 \pi}-10$

Mulher: $\mathrm{AMBC}\left(\mathrm{cm}^{2}\right)=\underline{[\mathrm{PB}(\mathrm{cm})-(\pi \times \mathrm{DCT}(\mathrm{cm}))]^{2}}-6,5$ $4 \pi$

Adicionalmente, a avaliação nutricional foi complementada pelo perfil bioquímico, sendo considerados os níveis de colesterol total, glicemia e albumina, determinados por meio de analisadores automáticos (Eclipse Vitalab; Merck, Holanda), e a dosagem de hemoglobina, realizada pelo método de contagem eletrônica, por meio do Contador Eletrônico Hematológico (Coulter Counter T- 890, Estados Unidos).

As seguintes variáveis foram entendidas como potenciais fatores de confusão no presente estudo: (a) características sociodemográficas (idade, sexo, escolaridade); (b) comportamentos em saúde (sedentarismo, tabagismo e consumo de álcool); (c) história de diagnóstico médico para hipertensão arterial.

Foram considerados sedentários os idosos com gasto energético avaliado pela taxa de equivalentes metabólicos (MET) menor que 450MET. $\mathrm{min} / \mathrm{semana}$, o que corresponde a pelo menos 150 minutos por semana de atividade física moderada a vigorosa ou intensa 16 . Foram considerados tabagistas atuais os idosos que relataram terem fumado 100 cigarros ou mais durante a vida e que eram fumantes na época da pesquisa. O consumo de bebida alcoólica nos 12 meses anteriores à entrevista foi considerado, independentemente da quantidade ou tipo de bebida ingerida.

As medidas de pressão arterial foram realizadas no início da manhã, depois de cinco minutos iniciais de repouso e após 30 minutos ou mais da ingestão de cafeína e/ou do uso de tabaco. Três medidas de pressão arterial foram obtidas com pelo menos dois minutos de intervalo, e a pressão arterial considerada foi a média da segunda e da terceira medida. A hipertensão arterial foi definida por pressão diastólica $\geq 90 \mathrm{mmHg}$ e/ou pressão sistólica $\geq 140 \mathrm{mmHg}$ e/ou uso atual de medicamentos para hipertensão arterial.

\section{Análise estatística}

Foi realizada uma análise descritiva, com percentual para variáveis categóricas, e média e desvio padrão para variáveis quantitativas. A comparação entre infectados pelo Tr. cruzi e não infectados foi realizada pelo teste t de Student, para variáveis contínuas, e pelo teste do qui-quadrado de Pearson, para variáveis categóricas.

A associação entre a infecção pelo Tr. cruzi e as variáveis nutricionais foi avaliada pelo cálculo das razões de prevalência e respectivos intervalos de 95\% de confiança (IC95\%), utilizando a regressão de Poisson com variância robusta. Essa análise foi ajustada pelos potenciais fatores de confusão pesquisados. Para essa análise, as variáveis nutricionais foram categorizadas segundo decis.

Todas as análises foram realizadas com uso do programa Stata 10.1 (Stata Corp., College Station, Estados Unidos), considerando a população total e estratificada por faixa etária (60-69 e 70 anos ou mais).

\section{Resultados}

Entre os participantes do estudo (1.479), 563 $(38,1 \%)$, apresentaram sorologia positiva para infecção pelo Tr. cruzi, sendo 31,1\% entre homens e $42,5 \%$ entre as mulheres ( $p<0,001)$. A Tabela 1 mostra as características dos participantes do estudo, segundo resultado da sorologia. A média de idade foi de 69,3 anos (DP $=7,4$ ), 60,9\% eram mulheres, $35,6 \%$ tinham 4 anos ou mais de escolaridade, $29,8 \%$ foram considerados sedentários, $18,1 \%$ eram fumantes atuais, $21,1 \%$ relataram consumo de bebida alcoólica nos últimos 12 meses e $61,4 \%$ eram hipertensos. Diferenças significativas em relação à sorologia para Tr. cruzi foram observadas para as variáveis sexo e escolaridade. 
Características dos participantes do estudo, segundo sorologia para Trypanosoma cruzi (linha de base da coorte de idosos de Bambuí). Minas Gerais, Brasil.

\begin{tabular}{|c|c|c|c|c|}
\hline \multirow[t]{2}{*}{ Variáveis } & \multirow{2}{*}{$\begin{array}{c}\text { Total } \\
(\mathrm{N}=1.479)\end{array}$} & \multicolumn{2}{|c|}{ Sorologia } & \multirow[t]{2}{*}{ Valor de $p$ * } \\
\hline & & $\begin{array}{l}\text { Positiva } \\
(n=563)\end{array}$ & $\begin{array}{l}\text { Negativa } \\
(n=916)\end{array}$ & \\
\hline \multicolumn{5}{|l|}{ Características sociodemográficas } \\
\hline Idade (em anos) [média (DP)] & $69,3(7,4)$ & $69,5(7,1)$ & $68,8(7,2)$ & 0,080 \\
\hline Sexo feminino [\%] & 60,9 & 68,0 & 56,6 & $<0,001$ \\
\hline Escolaridade $\geq 4$ anos [\%] & 35,6 & 15,7 & 47,7 & $<0,001$ \\
\hline \multicolumn{5}{|l|}{ Comportamentos em saúde e hipertensão } \\
\hline Sedentarismo [\%] & 29,8 & 32,1 & 28,4 & 0,133 \\
\hline Tabagismo atual [\%] & 18,1 & 17,6 & 18,5 & 0,675 \\
\hline Consumo de bebida alcoólica nos 12 últimos meses [\%] & 21,1 & 19,4 & 22,1 & 0,220 \\
\hline Hipertensão arterial [\%] & 61,4 & 60,2 & 62,1 & 0,468 \\
\hline \multicolumn{5}{|l|}{ Perfil antropométrico } \\
\hline IMC $\left(\mathrm{kg} / \mathrm{m}^{2}\right)$ [média (DP)] & $25,1(4,9)$ & $24,4(5,0)$ & $25,6(4,8)$ & $<0,001$ \\
\hline $\mathrm{CC}(\mathrm{cm})[$ média (DP)] & $91,2(11,3)$ & $89,1(11,2)$ & $92,5(11,1)$ & $<0,001$ \\
\hline $\mathrm{DCT}(\mathrm{mm})[$ média (DP)] & $17,2(8,7)$ & $16,7(8,8)$ & $17,5(8,7)$ & 0,096 \\
\hline PB (cm) [média (DP)] & $28,1(4,1)$ & $27,2(4,2)$ & $28,6(3,9)$ & $<0,001$ \\
\hline $\mathrm{CMB}(\mathrm{cm})[$ média (DP)] & $41,8(11,3)$ & $39,6(10,8)$ & $43,2(11,4)$ & $<0,001$ \\
\hline $\mathrm{AMBc}\left(\mathrm{cm}^{2}\right)$ [média (DP)] & $34,0(10,8)$ & $32,0(10,4)$ & $35,1(10,9)$ & $<0,001$ \\
\hline \multicolumn{5}{|l|}{ Perfil bioquímico } \\
\hline Dosagem de albumina (g/dL) [média (DP)] & $4,6(0,5)$ & $4,6(0,6)$ & $4,6(0,5)$ & 0,861 \\
\hline Nível de hemoglobina (g/dL) [média (DP)] & $14,5(1,4)$ & $14,4(1,3)$ & $14,5(1,4)$ & 0,137 \\
\hline Colesterol total (mg/dL) [média (DP)] & $233,2(49,1)$ & $233,7(50,0)$ & $232,9(48,6)$ & 0,779 \\
\hline Glicemia (mg/dL) [média (DP)] & $108,5(43,3)$ & $104,5(30,6)$ & $111,0(49,3)$ & 0,005 \\
\hline
\end{tabular}

AMBc: área muscular do braço corrigida; CC: circunferência da cintura; CMB: circunferência muscular do braço; DCT: dobra cutânea tricipital; DP: desvio padrão; IMC: índice massa corporal; PB: perímetro do braço.

* Teste t de Student para variáveis contínuas e teste do qui-quadrado de Pearson para variáveis categóricas.

Em relação aos fatores nutricionais, os idosos infectados apresentaram, significativamente, menores valores para IMC, CC, PB, CMB, AMBc e glicemia.

A Tabela 2 apresenta os valores médios das variáveis antropométricas e bioquímicas estratificadas por faixa etária, segundo a infecção por Tr. cruzi. As variáveis antropométricas IMC, CC, $\mathrm{PB}, \mathrm{CMB}$ e $\mathrm{AMBc}$ apresentaram menores valores entre os idosos soropositivos nas duas faixas etárias ( $\mathrm{p}<0,05)$, enquanto a DCT apresentou menor valor ( $p=0,013$ ) entre os soropositivos apenas na faixa etária de 70 anos e mais. Em relação às variáveis bioquímicas, o nível de glicemia foi significativamente mais baixo entre os idosos com sorologia positiva ( $\mathrm{p}=0,017)$, mas apenas no grupo de 60-69 anos.

A Tabela 3 apresenta a associação entre a infecção pelo Tr. cruzi e as variáveis antropométricas e bioquímicas, ajustando por potenciais fatores de confusão. Todas as variáveis antropométri- cas apresentaram associação significativa com a infecção, evidenciando uma menor prevalência da infecção entre os idosos com maiores valores para as variáveis antropométricas. Essa associação foi consistente nas duas faixas etárias pesquisadas, salvo para a DCT, que apresentou associação significativa apenas entre os idosos mais velhos. De maneira geral, para cada aumento de uma unidade (decil) das variáveis antropométricas, a prevalência da infecção reduz da ordem de 4 a $8 \%$. As variáveis bioquímicas consideradas neste estudo não apresentaram diferenças significativas em relação à sorologia para Tr. cruzi.

\section{Discussão}

Os resultados mais importantes desse estudo se referem à análise da associação entre os aspectos nutricionais e a infecção pelo Tr. cruzi, que evidenciou um comprometimento significativo 
Tabela 2

Características dos participantes do estudo, segundo faixa etária e sorologia para Trypanosoma cruzi (linha de base da Coorte de Idosos de Bambuí). Minas Gerais, Brasil.

\begin{tabular}{|c|c|c|c|c|c|c|c|c|}
\hline \multirow[t]{2}{*}{ Variáveis } & \multicolumn{4}{|c|}{$60-69$ anos } & \multicolumn{4}{|c|}{70 anos ou +} \\
\hline & $\begin{array}{l}\text { Sorologia } \\
\text { positiva }\end{array}$ & $\begin{array}{l}\text { Sorologia } \\
\text { negativa }\end{array}$ & Total & $\begin{array}{c}\text { Valor de } \\
p^{*}\end{array}$ & $\begin{array}{l}\text { Sorologia } \\
\text { positiva }\end{array}$ & $\begin{array}{l}\text { Sorologia } \\
\text { negativa }\end{array}$ & Total & Valor de $p$ * \\
\hline \multicolumn{9}{|l|}{ Perfil antropométrico } \\
\hline IMC (kg/m²) & $24,9(4,8)$ & $25,9(5,0)$ & $25,6(4,9)$ & 0,004 & $23,6(5,2)$ & $25,0(4,5)$ & $24,4(4,9)$ & $<0,001$ \\
\hline $\mathrm{CC}(\mathrm{cm})$ & $89,5(10,6)$ & $92,4(11,1)$ & $91,4(11,2)$ & $<0,001$ & $88,6(11,6)$ & $92,6(11,1)$ & $91,0(11,5)$ & $<0,001$ \\
\hline $\mathrm{DCT}(\mathrm{mm})$ & $18,0(9,2)$ & $18,1(9,2)$ & $18,1(9,2)$ & 0,900 & $14,9(7,9)$ & $16,6(7,6)$ & $15,9(7,8)$ & 0,013 \\
\hline $\mathrm{PB}(\mathrm{cm})$ & $28,1(3,9)$ & $29,2(3,8)$ & $28,8(3,9)$ & $<0,001$ & $26,1(4,3)$ & $27,5(3,8)$ & $26,9(4,1)$ & $<0,001$ \\
\hline $\mathrm{CMB}(\mathrm{cm})$ & $41,0(10,6)$ & $44,9(11,8)$ & $43,5(11,5)$ & $<0,001$ & $37,7(10,7)$ & $40,5(10,1)$ & $39,4(10,4)$ & 0,002 \\
\hline $\operatorname{AMBc}\left(\mathrm{cm}^{2}\right)$ & $33,3(10,0)$ & $36,8(11,2)$ & $35,6(10,9)$ & $<0,001$ & $30,2(10,6)$ & $32,5(9,8)$ & $31,6(10,2)$ & 0,007 \\
\hline \multicolumn{9}{|l|}{ Perfil bioquímico } \\
\hline $\begin{array}{l}\text { Dosagem de albumina } \\
\text { (g/dL) }\end{array}$ & $4,6(0,5)$ & $4,6(0,5)$ & $4,6(0,5)$ & 0,920 & $4,5(0,6)$ & $4,5(0,5)$ & $4,5(0,5)$ & 0,605 \\
\hline $\begin{array}{l}\text { Nível de hemoglobina } \\
(\mathrm{g} / \mathrm{dL})\end{array}$ & $14,5(1,3)$ & $14,7(1,4)$ & $14,6(1,4)$ & 0,230 & $14,2(1,4)$ & $14,3(1,5)$ & $14,3(1,4)$ & 0,541 \\
\hline Colesterol total (mg/dL) & $235,5(51,6)$ & $234,2(47,9)$ & $234,7(49,3)$ & 0,695 & $231,3(47,8)$ & $231,0(49,8)$ & $231,1(48,9)$ & 0,955 \\
\hline Glicemia (mg/dL) & $104,7(29,9)$ & $112,5(54,1)$ & $109,7(46,9)$ & 0,017 & $104,0(31,5)$ & $108,7(40,9)$ & $106,9(37,4)$ & 0,154 \\
\hline
\end{tabular}

Nota: valores apresentados como média (desvio-padrão).

$\mathrm{AMBc}$ : área muscular do braço corrigida; CC: circunferência da cintura; CMB: circunferência muscular do braço; DCT: dobra cutânea tricipital;

IMC: índice massa corporal; PB: perímetro do braço.

* Teste t de Student.

Tabela 3

Associação entre fatores nutricionais e sorologia positiva para Trypanosoma cruzi, segundo faixa etária (linha de base da Coorte de Idosos de Bambuí). Minas Gerais, Brasil.

\begin{tabular}{|c|c|c|c|}
\hline \multirow[t]{2}{*}{ Variáveis (em decis) } & \multicolumn{3}{|c|}{$\mathrm{RP}(\mathrm{IC} 95 \%)$ * } \\
\hline & 60-69 anos & 70 anos ou + & Total \\
\hline \multicolumn{4}{|l|}{ Perfil antropométrico } \\
\hline $\mathrm{IMC}\left(\mathrm{kg} / \mathrm{m}^{2}\right)$ & $0,96(0,93-0,98) * *$ & $0,94(0,91-0,98) * *$ & $0,95(0,93-0,97)$ ** \\
\hline $\mathrm{CC}(\mathrm{cm})$ & $0,95(0,92-0,98) * *$ & $0,94(0,91-0,97) * *$ & $0,94(0,92-0,97) * *$ \\
\hline $\mathrm{DCT}(\mathrm{mm})$ & $0,96(0,93-1,00)$ & $0,92(0,88-0,96)$ ** & $0,94(0,92-0,97)$ ** \\
\hline $\mathrm{PB}(\mathrm{cm})$ & $0,95(0,92-0,98) * *$ & $0,93(0,90-0,97) * *$ & $0,94(0,92-0,96) * *$ \\
\hline $\mathrm{CMB}(\mathrm{cm})$ & $0,95(0,91-0,98)$ ** & $0,95(0,91-0,99)$ ** & $0,95(0,93-0,97)$ ** \\
\hline $\mathrm{AMBc}\left(\mathrm{cm}^{2}\right)$ & $0,95(0,92-0,98) * *$ & $0,95(0,91-0,99)$ ** & $0,95(0,93-0,97) * *$ \\
\hline \multicolumn{4}{|l|}{ Perfil bioquímico } \\
\hline Dosagem de albumina (g/dL) & $0,99(0,97-1,02)$ & $1,01(0,98-1,05)$ & $1,00(0,98-1,03)$ \\
\hline Nível de hemoglobina (g/dL) & $1,00(0,97-1,04)$ & $1,01(0,98-1,05)$ & $1,01(0,98-1,03)$ \\
\hline Colesterol total (mg/dL) & $0,99(0,96-1,02)$ & $1,01(0,98-1,04)$ & $1,00(0,97-1,02)$ \\
\hline Glicemia (mg/dL) & $0,98(0,95-1,01)$ & $1,00(0,97-1,03)$ & $0,99(0,96-1,01)$ \\
\hline
\end{tabular}

AMBc: área muscular do braço corrigida; CC: circunferência da cintura; CMB: circunferência muscular do braço; DCT: dobra cutânea tricipital; IC95\%: intervalo de 95\% de confiança; IMC: índice massa corporal; PB: perímetro do braço; RP: razão de prevalência.

* Regressão de Poisson: razões de prevalência ajustadas pelas variáveis idade, sexo, escolaridade, hipertensão arterial, tabagismo, sedentarismo e consumo de álcool;

** Valor de $\mathrm{p}<0,05$ 
de todas as medidas antropométricas entre os idosos com sorologia positiva, sendo consistente nas duas faixas etárias pesquisadas. A única exceção foi a DCT que apresentou associação significativa somente entre os idosos mais velhos.

Em relação ao estado nutricional avaliado pela antropometria, estudo desenvolvido em 33 pacientes com megacólon demonstrou um elevado percentual de desnutrição proteico-calórica (64\%) 17 enquanto outro estudo conduzido entre 27 adultos com diagnóstico de megaesôfago mostrou um percentual semelhante para baixo peso (63\%), avaliado pelo IMC 18 . Entre escolares de 7 a 12 anos, um estudo caso-controle encontrou maior chance de déficit de peso $(\mathrm{OR}=$ 2,8; IC95\%: 1,4-5,6) e déficit de altura $(\mathrm{OR}=2,4$; IC95\%: 1,4-4,0) entre as crianças com sorologia positiva para Tr. cruzi, em comparação aos não infectados 19 . Por outro lado, a análise dos dados de 66 pacientes adultos com sorologia positiva para doença de Chagas, atendidos no ambulatório da Faculdade de Medicina de uma universidade brasileira, demonstrou elevado percentual de obesidade (62\%), avaliada pelo IMC, e de risco cardiovascular (55\%), avaliado pelo elevado valor da CC 20.

Em Bambuí, o baixo valor do IMC observado entre idosos chagásicos mostra um comprometimento nutricional desse grupo, embora se deva ressaltar que essa medida usada isoladamente pode não refletir de forma adequada o estado nutricional dos idosos, por não considerar a distribuição regional da gordura ${ }^{21}$. Nesse sentido, o presente artigo acrescenta por avaliar outros indicadores antropométricos que permitem uma melhor caracterização do perfil nutricional dos idosos chagásicos. Os menores valores observados para DCT entre os idosos com sorologia positiva podem indicar menor reserva de tecido adiposo, e essa associação só foi encontrada entre os indivíduos com 70 anos ou mais, reforçando a redução da massa gorda verificada com o processo de envelhecimento 22 . Os idosos chagásicos também apresentaram valores significativamente menores para $\mathrm{PB}, \mathrm{CMB}$ e $\mathrm{AMBc}$, indicando perda de massa muscular que, apesar de ser um fato esperado com o aumento da idade 22 fica evidente que tal declínio ocorre de forma mais acentuada entre idosos infectados pelo Tr. cruzi.

A CC é um indicador importante de ser avaliado em populações idosas, uma vez que a centralização da gordura corporal parece melhor predizer as complicações nessa população 23. Por outro lado, o baixo valor de tal medida observado entre os idosos chagásicos deve ser analisado com cautela, pois pode sugerir que esses indivíduos apresentam menor risco de de- senvolver doenças metabólicas quando comparados ao grupo com sorologia negativa para $T r$. cruzi. Portanto, levando-se em consideração que a forma cardíaca é a mais grave e frequente manifestação da doença de Chagas crônica ${ }^{2}$, esta medida, se utilizada isoladamente, é inadequada à avaliação de risco cardiovascular de idosos com a infecção crônica por Tr. cruzi porque pode estar refletindo o pior estado nutricional dessa população.

As variáveis bioquímicas são utilizadas na complementação da avaliação nutricional, todavia não apresentaram associação significativa com a doença de Chagas no presente estudo. Em estudo desenvolvido entre mulheres com 40 anos ou mais de idade atendidas no ambulatório de um hospital universitário, os níveis de glicemia e o diagnóstico de diabetes não foram associados com a infecção pelo Tr. cruzi 24 , de forma semelhante ao constatado no presente estudo. Por outro lado, estudo anterior demonstrou que a doença de Chagas crônica foi responsável pela maior velocidade de transporte e utilização das lipoproteínas circulantes, levando a menores níveis de lipídeos 25, o que não foi observado em Bambuí. Em relação ao perfil hematimétrico, estudo conduzido em 92 casos da doença não mostrou associação significativa entre a concentração de hemoglobina, ferro sérico e ferritina entre as diferentes formas clínicas da doença 26. Em relação aos níveis plasmáticos de albumina, estudos realizados em amostras menores também não observaram diferença significativa entre indivíduos com ou sem infecção pelo $T r$. cruzi 27,28 .

Deve-se considerar que o presente estudo mostra uma análise seccional dos dados coletados na linha de base da Coorte de Idosos de Bambuí, porém a relação causal entre as variáveis não pode ser estabelecida. Além disso, o estudo em população idosa deve considerar o viés de sobrevida, e os idosos com formas mais graves da doença de Chagas ou manifestações mais severas da desnutrição podem não estar representados nessa amostra. Por outro lado, ressalta-se que, pelo nosso conhecimento, esse é o primeiro estudo de base populacional desenvolvido entre idosos com elevada prevalência de infecção crônica por Tr. cruzi, que demonstrou um comprometimento significativo de diferentes variáveis antropométricas, o que sugere a concomitância da doença de Chagas crônica e pior estado nutricional, sendo esse resultado consistente nas duas faixas etárias pesquisadas. Além disso, a presente análise foi ajustada por potenciais fatores de confusão, que poderiam apresentar associação com a infecção pelo Tr. cruzi e com os indicadores nutricionais considerados. 
Em síntese, os resultados do presente estudo mostram a ocorrência simultânea da infecção crônica pelo Tr. cruzi e de importantes déficits nutricionais, incluindo redução da reserva de tecido adiposo e de massa muscular, em uma população idosa que viveu em área endêmica para doença de Chagas no passado. Esses resultados foram consistentes nas duas faixas etárias pesquisadas, demonstrando que a infecção crônica pelo Tr. cruzi pode estar ressaltando a redução das medidas antropométricas com a idade, que já é observada com o processo de envelhecimento. Logo, fica evidente a importância da avaliação nutricional entre idosos com infecção crônica, visando minimizar o potencial impacto da nutrição inadequada na evolução da doença nessa população.

\section{Resumen}

El objetivo del estudio fue verificar los aspectos nutricionales asociados a la infección crónica por el Trypanosoma cruzi entre los participantes de la línea de base de una cohorte de ancianos de Bambuí, Minas Gerais, Brasil. El análisis incluyó al 84,9\% (1.479) de todos los residentes con 60 años o más en la ciudad en 1997. La infección por el Tr. cruzi fue evaluada por tres testes serológicos y el perfil nutricional se caracterizó por variables antropométricas y bioquímicas. Las asociaciones se evaluaron por las razones de prevalencia e intervalos de un 95\% de confianza, utilizando la regresión de Poisson robusta y ajustada por potenciales factores de confusión. La infección se observó en un $38,1 \%$ de los ancianos. Todas las variables antropométricas presentaron una asociación significativa con la infección, evidenciando menores valores entre los ancianos con serología positiva. Las variables bioquímicas no se asociaron al evento estudiado. Los resultados evidenciaron la concomitancia de la enfermedad de Chagas crónica y un peor estado nutricional en esa población, reforzando la importancia de la evaluación nutricional entre ancianos con infección crónica por el Tr. cruzi.

Enfermedad de Chagas; Evaluación Nutricional; Anciano

\section{Colaboradores}

J. P. Santos participou da análise dos dados, interpretação dos resultados e redação do artigo. M. F. Lima-Costa colaborou no desenho do estudo, análise dos dados e revisão do manuscrito. S. V. Peixoto foi responsável pela supervisão da análise dos dados, discussão dos resultados, redação e revisão final do manuscrito.

\section{Agradecimentos}

O Projeto Bambuí recebeu financiamento do FINEP. M. F. Lima-Costa é bolsista de produtividade do CNPq. 


\section{Referências}

1. World Health Organization. Working to overcome the global impact of neglected tropical diseases. First WHO report on neglected tropical diseases. Geneva: World Health Organization; 2010.

2. Programa Especial de Investigaciones y Enseñanzas sobre Enfermedades Tropicales, Organización Mundial de la Salud. Reporte sobre la enfermedad de Chagas. http://www.who.int/tdr (acessado em 10/Ago/2012).

3. Rassi Jr. A, Rassi A, Marin-Neto JA. Chagas disease. Lancet 2010; 375:1388-402.

4. World Health Organization. Chagas disease (American trypanosomiasis) fact sheet (revised in June 2010). Wkly Epidemiol Rec 2010; 85:334-6.

5. Rassi Jr. A, Rassi A, Marcondes de Rezende J. American trypanosomiasis (Chagas disease). Infect Dis Clin North Am 2012; 26:275-91.

6. Gascon J, Bern C, Pinazo MJ. Chagas disease in Spain, the United States and other non-endemic countries. Acta Trop 2010; 115:22-7.

7. Lent H, Wygodzinsky P. Revision of the Triatominae (Hemiptera, Reduviidae), and their significance as vectors of Chagas disease. Bulletin of the American Museum of Natural History 1979; 163:123-520.

8. Departamento de Atenção Básica, Secretaria de Atenção à Saúde, Ministério da Saúde. Doença de Chagas. In: Secretaria de Atenção à Saúde, Ministério da Saúde, organizador. Vigilância em saúde: zoonoses. Brasília: Ministério da Saúde; 2009. p. 13-45. (Cadernos de Atenção Básica, 22) (Série B. Textos Básicos de Saúde).

9. Lima-Costa MF, Barreto SM, Guerra HL. Chagas' disease among older adults: branches or mainstream of the burden Trypanossoma cruzi infection. Int J Epidemiol 2002; 31:688-95.

10. Werneck GL, Hasselmann MH, Gouvêa TG. Panorama dos estudos sobre nutrição e doenças negligenciadas no Brasil. Ciênc Saúde Coletiva 2011 16:39-62.

11. Barreto SM, Passos VM, Lima-Costa MF. Obesity and underweight among Brazilian elderly. The Bambuí Health and Aging Study. Cad Saúde Pública 2003; 19:605-12.

12. Lima-Costa MF, Firmo JO, Uchoa E. Cohort profile: the Bambui (Brazil) Cohort Study of Ageing. Int Epidemiol 2011; 40:862-7.

13. Jelliffe DB. The assessment of nutrition states of the community. Geneva: World Health Organization; 1996.

14. Gurney JM, Jelliffe DB. Arm anthropometry in nutritional assessment: nomogram for rapid calculation of muscle circumference and cross-sectional muscle and fat areas. Am J Clin Nutr 1973; 26:912-5.

15. Heymsfield SB, McManus C, Smith J, Stevens V, Nixon DW. Anthropometric measurement of muscle mass: revised equations for calculating bone-free arm muscle area. Am J Clin Nutr 1982; 36:680-90.

16. Ramalho JRO, Lima-Costa MF, Firmo JOA, Peixoto SV. Energy expenditure through physical activity in a population of community-dwelling Brazilian elderly: cross-sectional evidences from the Bambuí cohort study of aging. Cad Saúde Pública 2011; 27 Suppl 3:S399-408.
17. Vieira MJ, Gama-Rodrigues JJ, Habr-Gama A, Faintuch J, Waitzberg DL, Pinotti HW. Preoperative assessment in cases of adult megacolon suffering from moderate malnutrition. Nutrition 1996; 12:491-5.

18. Penhavel FA, Waitzberg DL, Trevenzol HP, Alves L Zilberstein B, Gama-Rodrigues J. Pre-and postoperative nutritional evaluation in patients with chagasic megaesophagus. Nutr Hosp 2004; 19:89-94.

19. Andrade AL, Zicker F. Chronic malnutrition and Trypanosoma cruzi infection in children. J Trop Pediatr 1995; 41:112-5.

20. Geraix J, Ardisson LP, Marcondes-Machado J, Pereira PC. Clinical and nutritional profile of individuals with Chagas disease. Braz J Infect Dis 2007; 11:411-4

21. Lopes AC, Caiaffa WT, Sichieri R, Mingoti AS, Lima-Costa MF. Estado nutricional: antropometria consumo alimentar e dosagens bioquímicas de adultos e idosos - Projeto Bambuí um estudo de base populacional. REME Rev Min Enferm 2008; 12:483-93.

22. Kuczmarski MF, Kuczmarski RJ, Najjar M. Descriptive anthropometric reference data for older Americans. J Am Diet Assoc 2000; 100:59-66.

23. Visscher TL, Seidell JC, Molarius A, van der Kuip D, Hofman A, Witteman JC, et al. A comparison of body mass index, waist-hip ratio and waist circumference as predictors of all-cause mortality among the elderly: the Rotterdam study. Int J Obes Relat Metab Disord 2001; 25:1730-5.

24. Santos VM, Cunha SF, Teixeira VP, Monteiro JP, Santos JA, Santos TA, et al. Frequência de diabetes mellitus e hiperglicemia em mulheres chagásicas e não chagásicas. Rev Soc Bras Med Trop 1999; 32:489-96.

25. Diament J, Forti N, Giannini SD. Alterações metabólicas na doença de Chagas. Modernos conhecimentos sobre doença de Chagas. In: Suplemento dos Anais de 1979 e 1980 da Academia Mineira de Medicina. Belo Horizonte: Universidade Federal de Minas Gerais, Academia Mineira de Medicina; 1981. p. 115-28.

26. Calderoni DR, Andrade TS, Grotto HZ. Haptoglobin phenotype appears to effect the pathogenesis of American trypanosomiasis. Ann Trop Med Parasitol 2006; 100:213-21.

27. Pereira MG, Dórea JG, Johnson NE, Castro CN, Macêdo V. Serum albumin and gamma globulin in Trypanosoma cruzi infections. Trans R Soc Trop Med Hyg 1983; 77:32-4.

28. Cetron MS, Basilio FP, Moraes AP, Sousa AQ, Paes JN, Kahn SJ, et al. Humoral and cellular immune response of adults from Northeastern Brazil with chronic Trypanosoma cruzi infection: depressed cellular immune response to T. cruzi antigen 39 among Chagas' disease patients with symptomatic versus indeterminate infection. Am J Trop Med Hyg 1993; 49:370-82.

Recebido em 22/Ago/2012

Versão final reapresentada em 08/Jan/2013

Aprovado em 28/Jan/2013 